\title{
Carbon Nanotubes: Continued Innovations and Challenges
}

\section{M.S. Dresselhaus and H. Dai, Guest Editors}

\begin{abstract}
This article outlines the content of the April 2004 issue of MRS Bulletin on Advances in Carbon Nanotubes. Essentially, carbon nanotubes are self-assembling nanostructures constructed of sheets of hexagonal-shaped carbon atoms rolled up into cylinders. Carbon nanotubes have attracted a great deal of attention as model systems for nanoscience and for potential applications. The special interest in carbon nanotubes stems from their unique structure and properties: their very small size (down to $\sim 0.42 \mathrm{~nm}$ in diameter); the possibility for carbon nanotubes to be metallic or semiconducting, depending on their geometrical structure; their exceptional properties of ballistic transport; their extremely high thermal conductivity and high optical polarizability and the possibilities of high structural perfection. Research in the carbon nanotube field has now advanced to the stage where a good understanding of the structure and many of the basic properties are in place, together with much appreciation of their interrelation. On the other hand, major gaps in basic knowledge remain, with the major obstacles confronting the carbon nanotube field being the lack of a detailed understanding of the nanotube growth mechanism and control of the synthesis process to produce nanotubes with a desired diameter and chirality. The brief review of the carbon nanotube field by leading experts in this issue comes at an opportune time. Many exciting results on the structural, electronic, optical, and transport properties of these tiny well-ordered structures have already been achieved, and the research is well enough developed to assess present progress and identify new research directions waiting to be explored.
\end{abstract}

Keywords: ballistic transport, carbon nanotubes, chemical functionalization, chirality, field-effect transistors, fluorescence, photophysics, Raman spectroscopy.

Carbon nanotubes, as self-assembling nanostructures, have attracted a great deal of attention as model systems for nanoscience and for various potential applications, including composite materials, battery electrode materials, field emitters, nanoelectronics, and nanoscale sensors. The interest in carbon nanotubes stems from their unique structure and properties, including their very small size (down to $\sim 0.42 \mathrm{~nm}$ in diameter); the possibility for carbon nanotubes to be metallic or semiconducting, depending on their geometrical structure; their exceptional properties of ballistic transport; their extremely high thermal conductivity and optical polarizability; and the possibilities of high structural perfection.

Single-walled carbon nanotubes (SWNTs) have only one atomic species (carbon) and a relatively simple structure-a sheet of regular hexagons rolled in a seamless way into a cylinder one atom thick. Because of this simplicity, detailed calculations of the unique properties of SWNTs can be carried out. In fact, theoretical predictions that SWNTs can be either semiconducting or metallic, depending on their geometrical structure, were made ${ }^{1-3}$ before a SWNT was ever synthesized in the laboratory. 4,5

From the inception of research on SWNTs, they have served as a model one- dimensional (1D) system for a variety of new phenomena, where theory and experiment have worked hand-in-hand in advancing the field. SWNTs thus provide a benchmark for new phenomena that might be possible, based on their unique molecular density of states, whereby every structurally distinct $(n, m)$ nanotube (where $n$ and $m$ are the integers defining the wrapping vectors that describe the geometry of each tube) has its own unique and characteristic 1D density-of-states spectra. Now that nanotubes have been synthesized from many other chemical species including carbon, boron nitride, bismuth, metal chalcogenides, and many others, a wide range of novel properties may be discovered. Investigating how the properties of 1D nanotubes differ from 1D nanowires, 2D thin films, or 3D bulk structures of the same material should provide interesting and useful data.

Research in the carbon nanotube field has now advanced to the stage where a good understanding of the structure and of many of the basic properties is in place, together with an appreciation of their interrelation. Many unexpected phenomena that do not occur in the parent graphite material have been discovered in nanotubes, and these discoveries have energized not only nanotube research, but also nanoscience research in general. On the other hand, major gaps in our basic knowledge remain, with the major obstacle confronting the field being the lack of a detailed understanding of the nanotube growth mechanism. Such an understanding is needed because of the unusually close connection between nanotube properties (such as their metallicity) and their geometric structure; the knowledge will allow the diameter and chirality of nanotubes to be controlled by chemical synthesis methods.

The brief review by leading experts of the carbon nanotube field in this issue of the MRS Bulletin comes at an opportune time. Many exciting results regarding the structural, electronic, optical, and transport properties of these tiny well-ordered structures have already been achieved, and the research is well-enough developed to assess present progress and identify new research opportunities.

From the beginning, the main emphasis of carbon nanotube research has been in the synthesis area, and this remains the great challenge of the field. This viewpoint is strongly reflected in the organization of this issue. Rapid progress is being made to increase control of the synthesis process, steadily narrowing the diameter and chirality range of the nanotubes, decreasing defects and impurities, and in- 
creasing production efficiency and yield while expanding functionality. The main pursuits in controlling nanotube synthesis include the synthesis of molecular catalytic clusters with atomically well-defined size and shape, the development of mild, reduced-temperature catalytic synthesis conditions, the development of patterned growth with a high degree of control in nanotube location and orientation, and the synthesis of complex and organized networks or arrays of nanotubes on large substrates. Progress in the synthesis area and opportunities for new research are presented in the article by Liu et al.

Because SWNTs are usually grown in the presence of a variety of carbon species, catalyst particles, and other unwanted constituents, much attention has been given to nanotube purification. This has led to techniques for the characterization of nanotube purity, and the preparation/sorting of nanotubes by length, diameter, chirality, and metallic/semiconducting properties. These issues are discussed in detail in the article by Haddon et al.

The issues of synthesis and separation discussed in the articles by Liu et al. and Haddon et al. are closely related. Together, these articles reveal the approaches that eventually might lead to ultimate control of the nanotube synthesis process. With improved synthesis regarding purity and monodispersity in tube diameter and chirality, problems in purification and separation could be largely eliminated or avoided. On the other hand, if efficient and precise purification and separation techniques can be developed, challenges to growth can become much more tractable. It is highly likely that the convergence or combination of the two areas-that is, the controlled or preferential growth of a nanotube with a particular chirality, and purification/separation-could prove powerful in producing nanotubes with well-defined diameters and chiralities.

The filling of nanotubes with various fullerene species offers an entree into using nanotubes as a template for the synthesis of many new nanostructured species. Furthermore, the subsequent thermal conversion of the encapsulated fullerenes to an inner carbon nanotube gives rise to doublewalled carbon nanotubes (DWNTs), which are a prototype for studying the structure and properties of multiwalled carbon nanotubes (MWNTs) in a quantitative way. Because of the increased stability and durability of DWNTs and MWNTs relative to SWNTs, these more robust nanotubes have greater potential for applications requiring the exceptional mechanical strength, stiffness, and high thermal conductivity of carbon nanotubes. An assess- ment of current knowledge about the two prototype materials, SWNTs filled with fullerenes (called peapods) and DWNTs, is presented in the article by Bandow et al.

The use of nanotubes as a laboratory for producing novel nanostructures, including nanowires, is discussed in the article by Sloan et al. The spatial and structural constraints of the hollow core and innershell "surface" of the nanotube can give rise to unusual structures. Great strides have been made in both the synthesis of the novel structures within the cores of nanotubes and their detailed structural characterization by high-resolution transmission electron microscopy. Much remains to be done in investigating the unique physical properties possessed by these nanowires, how these properties might relate to their novel nanowire structures, and how the structure and properties of these nanowires relate to those of bulk 3D materials. The wide range of materials that have been shown to form endohedral nanowires within SWNTs is impressive, including the case of peapods containing fullerenes that merge to form a second nanotube within SWNTs. These peapods have thus far received the most attention, with many publications on their novel structural, electronic, and vibrational properties, as described in a complementary way in the articles by Sloan et al. and by Bandow et al. These results are just harbingers of the richness of new phenomena that are expected to occur under the unusual nanolaboratory conditions found within the core region of nanotubes.

Regarding property measurements, the most attention thus far has been devoted to studies of the remarkable transport properties observed in individual field-effect transistors (FETs), including ballistic transport, the suppression of carrier backscattering, single-electron transistor effects, enormously high current densities, good FET device performance, and the demonstration of a variety of device functions (AND and NOR gates, etc.). While high mobility and ballistic transport are partly due to the structural perfection possible with nanotubes, the chemical stability and robustness of nanotubes and the lack of unsaturated surface dangling bonds make nanotubes unique among electronic materials. Sustaining ultrahigh current flows and retaining high mobility are possible when nanotubes are processed and integrated into realistic transistor structures. Recent achievements in advancing our understanding of the remarkable electron transport properties of both semiconducting and metallic single-walled nanotubes are described in the article by McEuen and Park.
The optical photophysics of nanotubes has likewise received considerable attention as a means for studying electron, phonon, and optoelectronic phenomena. Progress on this topic and future challenges are presented in the article by Jorio et al. Since Raman scattering and optical absorption and emission in SWNTs are resonant processes, depending on the singularities in the 1D density of electronic states, these techniques provide a convenient method for characterizing the diameter distribution and the metallicity distribution of the SWNTs in a sample. The relative sensitivities and resolution of the various techniques, such as Raman scattering, optical luminescence, and optical absorption are discussed, although the emphasis in this article is on the new physics uncovered through these studies. Fast optics provides unique information about lifetimes of excited states, while photophysics and transport properties merge in areas of electroluminescence and photoconductivity, two frontiers of nanotube photophysics at present.

A great amount of effort and emphasis in nanotube research has focused on the eventual use of carbon nanotubes in devices. The article by de Heer presents a critical evaluation of progress in the commercial exploitation of carbon nanotubes. Most researchers in the nanotube field at universities, industrial research laboratories, and government laboratories worldwide are optimistic about important and even large-scale applications eventually emerging from nanotube research, although at present the largest volume applications use MWNTs for increasing the strength and modulus of composite materials, and for improving the lifetime and performance of lithium-ion batteries. There are laboratory demonstrations of many interesting prototype devices, but much of the actual commercialization remains in the future. Almost all of the articles allude to applications of nanotubes and to their future commercial exploitation.

The future of nanotubes for electronics applications remains bright. The nearly unparalleled and unprecedented electrical properties and chemical stability of nanotubes provide a powerful driving force to fully utilize these properties. Nanotubes are just beginning to be exploited for building high-performance transistors, but their use has yet to be convincingly demonstrated for interconnect applications. Many device designers are eagerly awaiting breakthroughs to enable them to interconnect increasing numbers of devices in smaller spaces. Nanotubes offer great promise here. Applications of nanotubes as fluorescence-label-free electronic, chemi- 
cal, and biological sensors, especially in miniaturized, multiplexed, and arrayed forms, are novel and promising. For sensor applications, much less stringent requirements will be placed on the nanotube chirality, and just like other sensor technologies, the challenges lie in selectivity and strategies in multiplexing, arraying, and addressing. In the past several years, there has been a steady increase in activities that incorporate nanotubes into biological systems, including proteins, DNA, and living cells. This is an interesting and relatively new area that can be categorized as the "wet" side of nanotube science and technology. Exciting results and discoveries have already been achieved in nanotube research, and much more can be expected in the next few years.

\section{References}

1. R. Saito, M. Fujita, G. Dresselhaus, and M.S Dresselhaus, Phys. Rev. B 46 (1992) p. 1804.

2. J.W. Mintmire, D.H. Robertson, and C.T.
White, J. Phys. Chem. Solids 54 (1993) p. 1835.

3. R. Saito, G. Dresselhaus, and M.S. Dresselhaus, Physical Properties of Carbon Nanotubes (Imperial College Press, London, 1998).

4. S. Iijima and T. Ichihashi, Nature 363 (1993) p. 603 .

5. D.S. Bethune, C.H. Kiang, M.S. de Vries, G. Gorman, R. Savoy, J. Vazquez, and R. Beyers, Nature 363 (1993) p. 605.

\section{Mildred S. Dresselhaus, Guest Editor for this issue of MRS Bulletin, is Institute Professor of physics and electrical engineering at the Massachusetts Institute of Technology. She has been active in research across broad areas of solid-state physics, especially in carbon science. Her present research activities focus on nanoscience, with special emphasis on carbon nanotubes,} bismuth nanowires, low-dimensional thermoelectricity, and novel forms of carbon.

Dresselhaus received her PhD degree in physics from the University of Chicago in 1958. She joined the MIT faculty in 1967 and has been an Institute Professor since 1985. She has received the $\mathrm{Na}$ tional Medal of Science and 19 honorary degrees worldwide. Dresselhaus also served as the director of the Office of Science at the U.S.

Department of Energy in 2000-2001.

Dresselhaus can be reached by e-mail at millie@mgm.mit.edu.

Hongjie Dai, Guest Editor for this issue of MRS Bulletin, is an associate professor in the Chemistry Department at Stanford University. His research spans the areas of nanomaterials synthesis, electron transport, molecular electronics, and chemical and biological sensors.

Dai received his bachelor's degree from Tsinghua University in China in 1989 and his $\mathrm{PhD}$ degree in physical chemistry and applied sciences from Harvard University in 1994; he carried out postdoctoral research at Rice University from 1994 to 1997 before joining the faculty at Stanford.

Dai can be reached by e-mail at hdai@stanford. edu.

Shunji Bandow is an associate professor in the Department of Materials Science and Engineering at Meijo University in Nagoya, Japan. His primary research interests have been in the fields of materials science (especially in finite-sized materials) and physics. $\mathrm{He}$ is experienced in electron microscopy, electron spin resonance, magnetic measurement, and Raman scattering, and has studied the quantum size effect of pure metal nanoparticles, metallofullerenes, and nanotubes.

Bandow received his BS degree in electric engineering from Meijo University in 1981 and his doctorate in science from the Tokyo Institute of Technology in 1991. Prior to joining the faculty of Meijo University in 2001, he was a technical associate at the Insti-

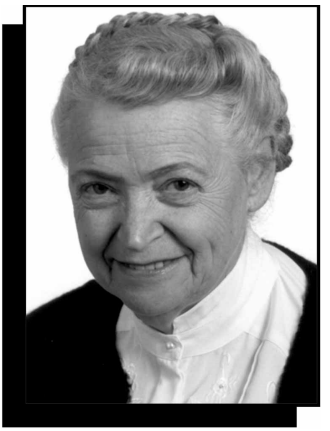

Mildred S. Dresselhaus

tute for Molecular Science, a research associate in the Instrument Center and the Center for Molecular Materials at the Institute for Molecular Science, and a research associate with the Nanotubulites Project of the Japan Science and Technology Corporation. He was also a visiting scientist at the University of Kentucky. Bandow has authored and co-authored more than 80 papers on a wide range of materials science topics.

Bandow can be reached by e-mail at bandow@ ccmfs.meijo-u.ac.jp.

Gugang Chen is a postdoctoral researcher in the Physics Department at the Pennsylvania State University. His areas of research include optical studies of nanomaterials (carbon nanotubes, semiconductor nanowires, etc.), chemical doping of nanotubes, and gas adsorption on these quantum filaments. He is currently

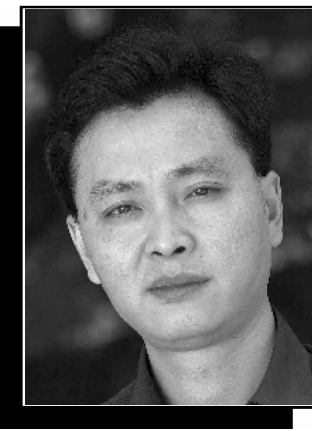

Hongjie Dai

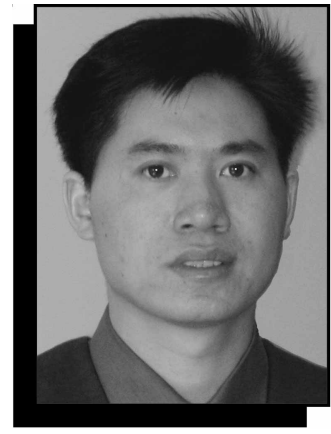

Gugang Chen

developing lithographic procedures for making electrical contacts to individual nanotubes and nanowires.

Chen received a BS degree (1994) in semiconductor physics and devices and a MS degree (1997) in condensedmatter physics from Wuhan University (China), working on the synthesis, purification, and optical properties of fullerene materials. Chen received his $\mathrm{PhD}$ degree from Penn State in 2003, where he focused on the optical properties of nanotubes.

Chen has authored more than 10 peer-

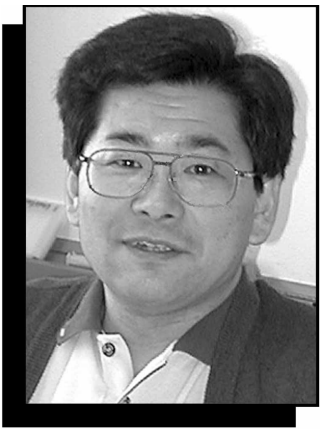

Shunji Bandow

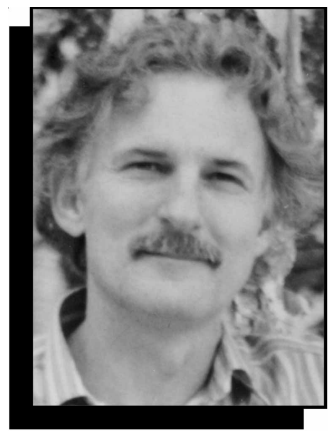

Walt A. de Heer

reviewed research papers and 20 international conference presentations. He received the Duncan fellowship from Penn State in 2002 and 2003. Chen is a member of the American Physical Society (APS) and the Material Research Society (MRS).

Chen can be reached by e-mail at gxc168@ psu.edu.

Walt A. de Heer is a professor in the School of Physics at the Georgia Institute of Technology. He joined the faculty in 1996 and has been engaged in research on nanotubes, leading to 
the discovery of roomtemperature ballistic conduction in carbon nanotubes in 1998. He developed a electronmicroscopy-based resonance method to measure the elastic properties of nanotubes in 1999.

Recently, he has found evidence for superconductivity in small niobium clusters. Currently, he is working on carbon nanotubes, ultrathin patterned graphite films, and the electronic and magnetic properties of cold metal clusters in molecular beams.

De Heer received his PhD degree at the University of California, Berkeley, in 1985. He investigated the properties of alkali clusters in molecular beams, resulting in the discovery of the electronic shell structure and plasma resonances. He moved to Lausanne, Switzerland, in 1987, where he worked at the EPFL until 1996. While there, he studied the magnetic properties of free transition-metal clusters. In 1992, he started his investigations of arc-produced multiwalled carbon nanotubes. Investigations of nanotube films cumulated in the discovery of their fieldemission properties.

De Heer can be reached by e-mail at deheer@ electra.physics.gatech.edu.

Gene Dresselhaus is a member of the senior staff within the Francis Bitter Magnet Laboratory at the Massachusetts Institute of Technology. His area of interest is the electronic structure of nanomaterials, and he has co-authored with M.S Dresselhaus several books on fullerenes, nanowires, and nanotubes.

Dresselhaus received his $\mathrm{PhD}$ degree in physics

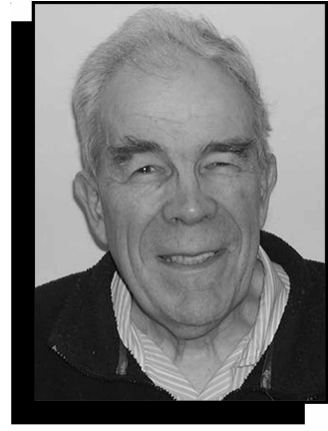

Gene Dresselhaus

from the University of California in 1955. He was a faculty member at the University of Chicago and an assistant professor at Cornell University before joining the MIT Lincoln Laboratory in 1960 as a staff member. In 1976, he assumed his current senior staff position.

Dresselhaus can be reached by e-mail at gene@mgm.mit.edu.

Peter C. Eklund is a professor of physics at the Pennsylvania State University. He is also associated with the Department of Materials Science and Engineering and the Penn State Materials Research Institute. Many of the materials he has made and studied have been built from carbon (e.g., diamond films, intercalated graphite, fullerenes, carbon nanotubes). His current research interests lie in the physics of quantum wires, nanoscale filaments, and related electronic devices. He maintains a general interest in materials synthesis, optical and transport properties, IR and Raman vibrational spectroscopy, hydrogen storage and generation, and chemical sensors.

Eklund received his undergraduate and graduate degrees in physics from the University of California,

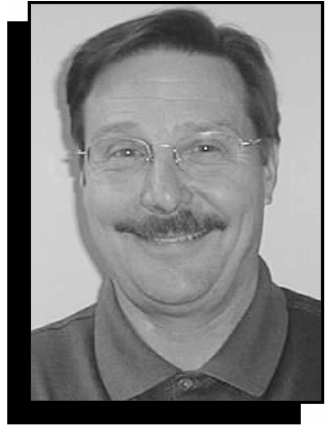

Peter C. Eklund

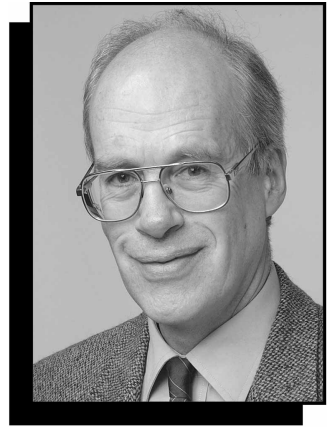

John L. Hutchison

Berkeley, and Purdue University, respectively, and he obtained his postdoctoral training at the Massachusetts Institute of Technology. He is a fellow of the American Physical Society and serves on the Solid-State Sciences Committee of the National Academy of Science. He is a coauthor of The Science of Fullerenes and Carbon Nanotubes (Academic Press, 1996) and a coeditor of Fullerene Polymers and Fullerene Polymer Composites (Springer Series on Materials Science, 2000). He cofounded two R\&D businesses, both of which are still active (NeoPhotonics in Fremont, Calif., and CarboLex in Lexington, Ky.).

Eklund can be reached by e-mail at pce3@psu.edu.

Shoushan Fan is a professor of physics, director of the TsinghuaFoxconn Nanotechnology Research Center,

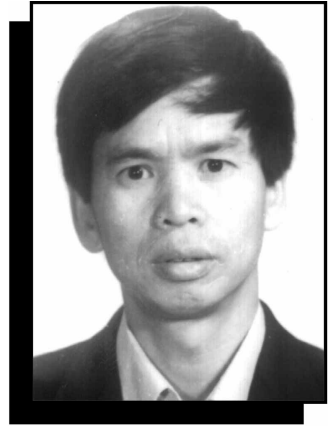

Shoushan Fan

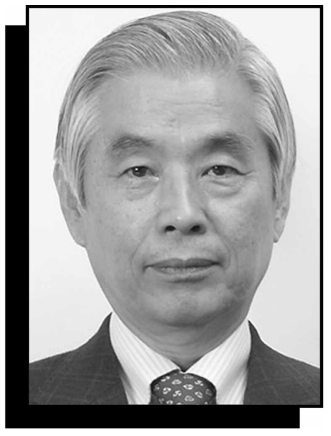

Sumio Iijima

and associate director of the Institute of Materials Science and Engineering at Tsinghua University in Beijing, China. His recent research interests are focused on the fabrication, characterization, and application of nanomaterials and nanodevices.

Fan graduated from Tsinghua University in 1981, then joined the faculty of the Physics Department. He has been a visiting scientist at the Massachusetts Institute of Technology,

Harvard University, and Stanford University.

Fan can be reached by e-mail at fss-dmp@ tsinghua.edu.cn.

Malcolm L.H. Green is head of the Inorganic Chemistry Department at the University of Oxford, where he has been since 1998. His research interests include organotransition-metal chemistry, homogeneous and heterogeneous catalysts for methane conversion

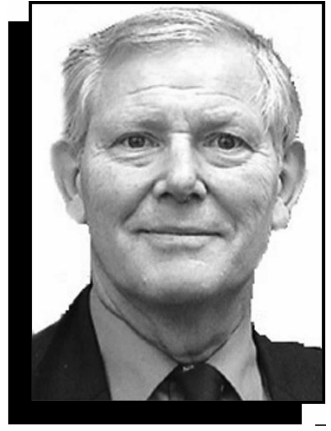

Malcolm L. H. Green

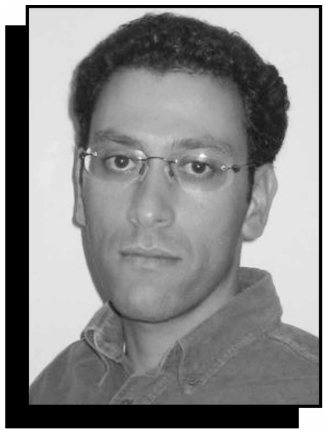

Ado Jorio

chemistry, and the chemistry of carbon nanotubes.

He received his $\mathrm{PhD}$ degree from the Imperial College of Science and Technology. Among his many awards and honors are the Davy Medal of the Royal Society, A.D.

Little Lecturer of the

Massachusetts Institute of Technology, the American Chemical Society Award in Organometallic Chemistry, the Fred Basolo Medal and Lecture of Northwestern University, the Ernest H. Swift Lectureship of the California Institute of Technology, the Sir Geoffrey Wilkinson Medal and Prize from the Royal Society of Chemistry, the Lewis Lecture of Cambridge, and the FMC Lecturer of Princeton University.

He is a fellow of the Royal Society. He was named doutor honoris causa by the University of Lisbon, Portugal, and has been a Distinguished 


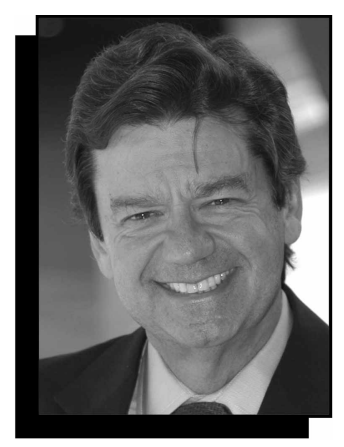

Robert C. Haddon

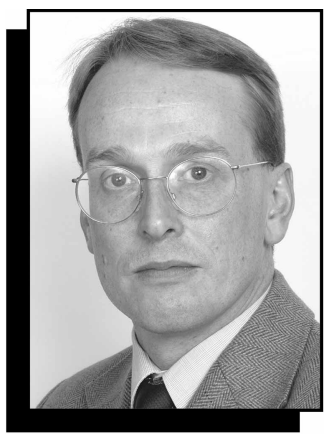

Angus I. Kirkland

Visiting Professor at Hong Kong University.

Green can be

reached by e-mail at malcolm.green@ chemistry.oxford.ac.uk.

\section{Robert C. Haddon} spent most of his career at Bell Laboratories (AT\&T, Lucent Technologies), where he was a Distinguished Member of Technical Staff in the Materials Chemistry Department. He joined the University of California, Riverside, in 2000 as a Distinguished Professor in the Departments of Chemistry and Chemical \& Environmental Engineering and as director of the Center for Nanoscale Science and Engineering (CNSE).

Haddon is best known for the prediction and discovery of superconductivity in alkali-metaldoped carbon-60. His research group is currently working on the chemistry and applications of carbon nanotubes and on the

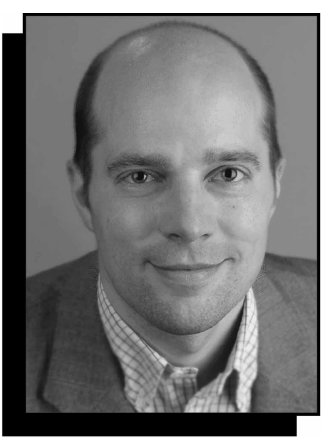

Tobias Hertel

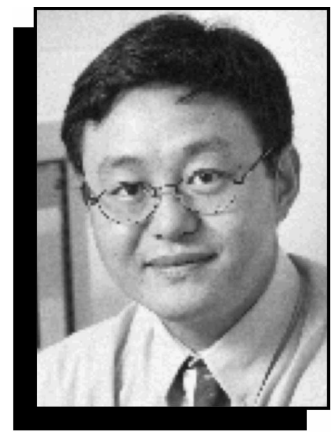

Jie Liu

synthesis and properties of neutral radical molecular conductors.

Haddon can be reached by e-mail at haddon@ucr.edu.

Tobias Hertel is an associate professor in the Department of Physics and Astronomy at Vanderbilt University in Nashville. His work concentrates on photostimulated processes at metal surfaces, carbon nanotubes, and other novel materials, with a particular focus on optical and electron spectroscopic studies of ultrafast dynamics.

Hertel received his $\mathrm{PhD}$ in physics from the Free University in Berlin in 1995. He became a faulty member at Vanderbilt University in 2004, where he is also co-director at the Vanderbilt Institute of Nanoscale Science and Engineering. He organized the Nanotube 2001 International Workshop and is organizer of the

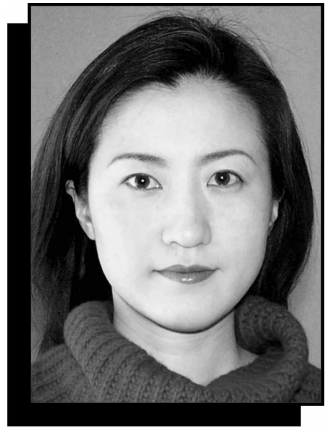

Kaori Hirahara

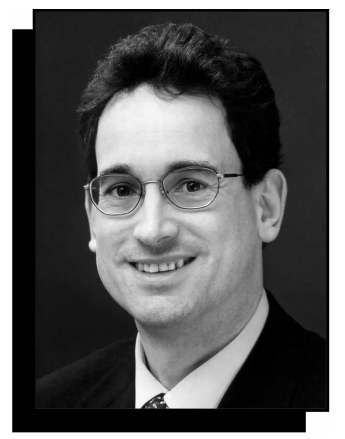

David E. Luzzi

first International Workshop on the Spectroscopy of Nanotubes in 2005. Among his honors are the Feodor

Lynen Scholarship from the Alexander von Humboldt Foundation, the Carl Ramsauer Award, and the Otto Hahn Medal from the Max Planck Society.

Hertel can be reached by e-mail at tobias. hertel@vanderbilt.edu.

Kaori Hirahara is a researcher in the Department of Materials Science and Engineering at Meijo University in Nagoya, Japan. Her primary research interests are in the fields of materials science in finite-sized materials and physics. She is experienced in highresolution transmission electron microscopy techniques (especially nanodiffraction) and has studied structural characterizations of single- and multi-walled carbon nanotubes,

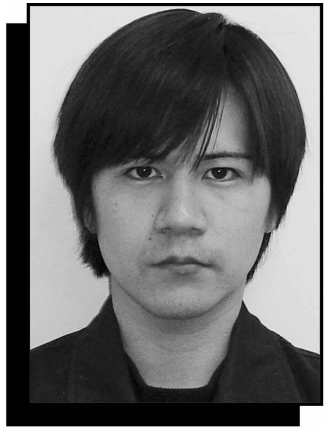

Tatsuki Hiraoka

metallofullerenes, and peapods.

Hirahara received her MS degree in applied physics from Nagoya University in 1999. Prior to joining Meijo University in 2003, she was a research associate with the Nanotubulites Project of the Japan Science and Technology Corporation.

Hirahara can be reached by e-mail at kaori_h@ccmfs.meijou.ac.jp.

Tatsuki Hiraoka is a doctoral student in the Department of Materials Science and Engineering at Meijo University in Nagoya, Japan. He is also a research fellow of the Japan Society for the Promotion of Science. He has investigated the synthesis of nanotubes by catalytic chemical vapor deposition, using transmission electron microscopy and Raman scattering.

Hiraoka earned a BS degree in materials science from Himeji Institute of Technology in 2001 and an MS degree in chemistry from Nagoya University in 2003.

Hiraoka can be reached at tatsuki@ ccmfs.meijo-u.ac.jp.

John L. Hutchison is a reader in materials at the University of $\mathrm{Ox}$ ford. His research inter- ests currently include the development of aberration-corrected high-resolution electron microscopy (HREM) techniques, structural disorder in complex oxides, nanostructured materials, and filled carbon nanotubes.

Hutchison obtained his BSc and PhD degrees at Glasgow University before moving to Oxford, where he set up and developed HREM facilities in the Inorganic Chemistry Laboratory. After a time at the University College of Wales in Aberystwyth, he returned to Oxford to take charge of the HREM facilities in the Department of Materials. In addition to his current position at Oxford, he is also president of the Royal Microscopical Society.

Hutchison can be reached by e-mail at john.hutchison@ materials.oxford.ac.uk.

Sumio Iijima is a professor in the Department of Materials Science and Engineering at Meijo University in Nagoya, Japan, and is also a parttime research fellow at the Fundamental Research Laboratories of NEC Corporation.

After receiving his PhD degree in solid-state physics from Tohoku University in 1969, Iijima worked as a research assistant in the Research Institute for Scientific Measurement at Tohoku University until 1970. He then moved to Arizona State University, where he stayed until 1982, except for a short leave at Cambridge University in 1979, working first as a postdoctoral associate and later as a senior research associate.

Iijima is a fellow of the American Physical Society and served as 
president of the Japanese Microscopy Society from 2001 to 2003. He was elected as a director of the Research Center for Advanced Carbon Materials at the National Institute of Advanced Industrial Science and Technology in Japan. Iijima can be reached by e-mail at iijimas@ ccmfs.meijo-u.ac.jp.

Ado Jorio is an associate professor in the Physics Department at the Universidade Federal de Minas Gerais (UFMG) in Belo Horizonte, Brazil. Jorio's research interests focus on resonance Raman techniques and on photophysics of nanostructures.

He joined the faculty of UFMG in 2002 after earning his $\mathrm{PhD}$ degree in physics there in 1999. He spent one year at ILL in Grenoble, working with phase transitions on incommensurate crystals, and two years as a postdoc at the Massachusetts Institute of Technology, working with Raman spectroscopy of carbon nanotubes.

Jorio was selected for the Profix fellowship program by the Brazilian agency CNPq in 2001.

$\mathrm{He}$ is a member of the Brazilian Nanoscience Institute.

Jorio can be reached by e-mail at adojorio@ fisica.ufmg.br.

Angus I. Kirkland is the Leverhulme Senior Research Lecturer in the Department of Materials at the University of Oxford. The current interests of his research group include the application of image processing to electron microscopy, the development of new detectors for high-energy electrons, aberration correction and measurement, and the design of elec- tron optical control systems. He is also actively involved in programs investigating the structures of nanocrystalline materials, particularly those encapsulated in carbon nanotubes.

Kirkland read natural sciences at Gonville and Caius College, Cambridge, graduating in 1986, prior to completing his PhD degree in highresolution electron microscopy and image simulation at Cambridge in 1989. After his PhD,

he worked in Cambridge as an SERC fellow (1989-1991), a British Ramsay Memorial research fellow (1991-1993), and a JEOL research associate (1993-1997) before being named a senior research fellow. He was appointed to his current position in 2002.

Kirkland is a fellow of the Royal Society of Chemistry, the Institute of Physics, and the Royal Microscopical Society. In 1992, he was elected a fellow of Fitzwilliam College, Cambridge, and in 2003 he was elected a fellow of Linacre College, Oxford.

Kirkland can be reached by e-mail at angus.kirkland@ materials.oxford.ac.uk.

Jie Liu is an assistant professor in the Chemistry Department at Duke University in Durham, North Carolina, where he has been on the faculty since 1999 . $\mathrm{He}$ received his $\mathrm{PhD}$ degree in chemistry at Harvard University in 1996 and was a postdoctoral research fellow at Rice University in Houston between 1996 and 1999. Liu's research group at Duke has focused on the synthesis of singlewalled carbon nanotubes using chemical vapor deposition and the de- velopment of scanningprobe-based lithographic techniques. He has published more than 20 papers over the last four years in the field of nanoscale material synthesis and functionalization.

Liu can be reached by e-mail at j.liu@duke.edu.

David E. Luzzi is a professor in the Department of Materials Science and Engineering at the University of Pennsylvania, where he has engaged in the scientific exploration of atomic-level structure and processes as a faculty member for 14 years. He is a Scientific Principal of NanoSelect Inc., a member of the Air Force Scientific Advisory Board, and an Editorial Board member of Applied Physics A. He is a director of the Nanotechnology Institute, in which he oversees the Institute's discovery and implementation activities in nano-biotechnology.

Luzzi's research program has produced over 100 publications and patents on nanoscale phenomena, instrumentation, and the synthesis, structure, properties, and application of materials. Luzzi's research group discovered the "peapod" class of functional nanomaterials, developing efficient synthesis routes, solving structures, and demonstrating unique properties. For this work,

Luzzi received the

George Heilmeier Award for Research Innovation. He has conducted a total of three years of research in Japan, is an alumnus of the IDA Defense Science Study Group, and was a science and technology fellow with the CNO Strategic Study Group.

Luzzi can be reached at luzzi@lrsm.upenn.edu.

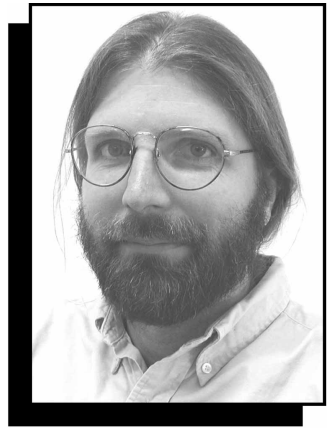

Paul L. McEuen

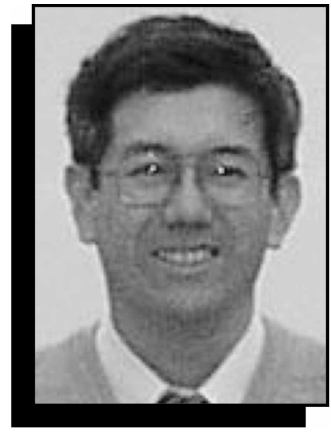

Riichiro Saito

Paul L. McEuen joined the faculty of Cornell University in 2001 as a professor of physics. His research examines the science and technology of nanostructures and has included studies of nanotubes, quantum dots, and single molecules. He also develops advanced measurement techniques to probe nanometer-scale systems.

McEuen received his BS degree in engineering physics from the University of Oklahoma in 1985 and his PhD degree in applied physics from Yale University in 1991. He was a postdoctoral researcher at the

Massachusetts Institute of Technology before going to the University of California, Berkeley, in 1992, where he was an assistant professor and later associate professor of physics and a researcher at Lawrence Berkeley National Laboratory. He is a fellow of the American Physical

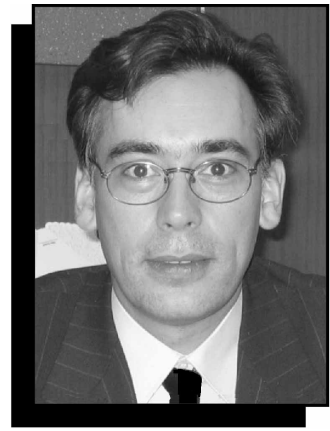

F. Papadimitrakopoulos

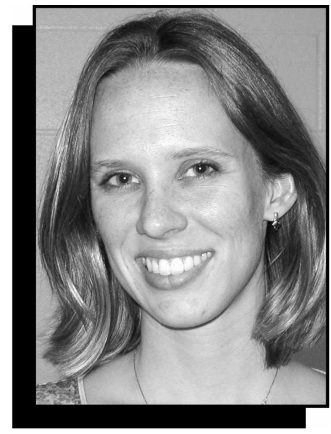

Jennifer Sippel

Society and recipient of the 2001 Agilent Europhysics Prize for work on carbon nanotubes.

McEuen can be reached by e-mail at mceuen@ ccmr.cornell.edu.

Fotios Papadimitrakopoulos is an associate professor of chemistry at the University of Connecticut. He also serves as the associate director of the Institute of Materials Science and co-director of the Nanomaterials Optoelectronics Laboratory at the university. The common theme of his research is self-organization; he has established a diverse research program that spans single-walled carbon nanotubes, CdSe and $\mathrm{Si}$ semiconductor nanoparticles, metalorganic chelates, and DNA assembly of colloidal microspheres. His most recent work focuses on the purification and separation of singlewalled carbon nano- 


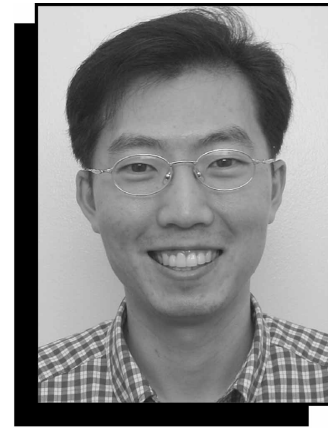

Ji-Yong Park

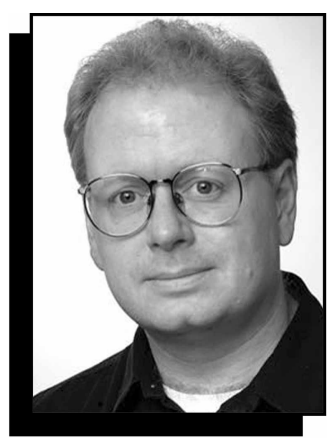

Jeremy Sloan

tubes by length, type, and diameter. He has co-authored more than 70 publications, given more than 100 invited presentations, and coorganized five international conferences.

Papadimitrakopoulos obtained his BS degree in chemistry at the University of Athens, Greece, and MS and PhD degrees from the Polymer Science and Engineering Department of the University of Massachusetts in 1989 and 1993, respectively. Before joining the University of Connecticut, he spent two years at AT\&T Bell Laborato-

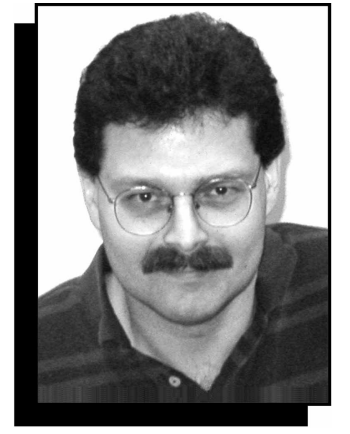

Andrew G. Rinzler

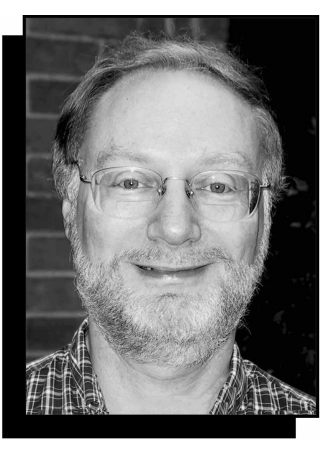

R. Bruce Weisman

ries as a postdoctoral member of technical staff.

Papadimitrakopoulos can be reached by e-mail at papadim@mail.ims. uconn.edu and Web site www.ims.uconn.edu.

Ji-Yong Park is a postdoctoral research associate in the Laboratory of Atomic and Solid-State Physics at Cornell University, where he works on electrical and mechanical properties of carbon nanotube devices through electrical transport measurements and scanning probe microscopy.

He received his MA (1995) and PhD (2000) degrees in physics from Seoul National University in Seoul, Korea. His main interests are transport properties of nanowires and nanotubes, nanoscale characterization of materials with scanning probe microscopy, and nanoelectronic devices.

Park can be reached by e-mail at jp276@ cornell.edu.

\section{Andrew G. Rinzler is} an associate professor of physics at the University of Florida. His research group was the first to experimentally confirm theoretical predictions about the polarizationdependence of light absorption by carbon nanotubes. More recently, they have devised a method for the separation of metallic and semiconducting nanotubes and have extended the functionality of nanotube-based atomic force microscopy probes.

Rinzler earned his $\mathrm{PhD}$ degree in physics at the University of Connecticut in 1991. He was a National Research Council postdoctoral fellow and also served as a postdoctoral researcher in the laboratory of Richard Smalley at Rice University.

Rinzler can be reached by e-mail at rinzler@ phys.ufl.edu.

Riichiro Saito is a professor in the Department of Physics at Tohoku
University in Japan. He received a $\mathrm{PhD}$ degree in physics from the University of Tokyo in 1985 and was a research associate there and an associate professor at the University of ElectroCommunication in Tokyo before joining the Tohoku faculty in 2003.

Saito is the author of Physical Properties of Carbon Nanotubes, published by Imperial College Press in 1998. He received the Japan IBM prize in 1999.

Saito can be reached by e-mail at rsaito@ flex.phys.tohoku.ac.jp.

Jennifer Sippel is a graduate student at the University of Florida, studying various electrical and mechanical properties of carbon nanotubes.

She earned her undergraduate degree in physics and astronomy from the University of Iowa.

Sippel can be reached by e-mail at sippicup@ phys.ufl.edu.

Jeremy Sloan is a senior research fellow in the Electron Microscopy and Microanalysis Group within the Department of Materials at the University of Oxford. He obtained his BSc degree in chemistry from the University of Hull in 1982, his MSc degree in 1990 from the State University of New York at Binghamton under the supervision of Professor Cliff Myers, and his $\mathrm{PhD}$ degree in materials in 1995 from the University of Wales, Cardiff, under the supervision of Professor Richard Tilley, FRSC.

Sloan joined the Inorganic Chemistry Laboratory, Oxford, in 1995 as a postdoctoral research assistant to Professor Malcolm Green, FRS. In 2000, he was awarded a Royal Society university research fellowship and in 2003 was made a fellow of Wolfson College, Oxford.

Sloan can be reached by e-mail at jeremy.sloan@ chem.ox.ac.uk.

R. Bruce Weisman is a professor of chemistry at Rice University in Houston and is a member of the Center for Nanoscale Science and Technology and the Center for Biological and Environmental Nanotechnology at Rice. Weisman's research interests focus on the spectroscopy and photophysics of carbon nanostructures.

He received his $\mathrm{PhD}$ in physical chemistry from the University of Chicago in 1977 and did postdoctoral research at the University of Pennsylvania. In 1979, he joined the Rice faculty.

Weisman can be reached by e-mail at weisman@rice.edu.

\section{Over 10,000 Proceedings Papers Now FREE Online For MRS Members}

\begin{tabular}{|c|c|}
\hline & $\begin{array}{l}\text { MRS members enjoy FREE access to the Online Proceedings Library-pr } \\
2000 \text { MRS Spring Meeting to the present. Plus free online access to M } \\
\text { Visit the MRS Web site at www.mrs.org and click on "Members Only" } \\
\text { Not a member? Join Today at www.mrs.org/membership/ }\end{array}$ \\
\hline
\end{tabular}

